\title{
Die politische Regulierung globaler Finanzrisiken
}

Susanne Lütz

\section{$1 \quad$ Einleitung}

Nirgendwo sonst ist die Marktexpansion so weit fortgeschritten wie im Finanzsektor. Fortschritte in der Informations- und Kommunikationstechnik, politisch motivierte Deregulierungsmaßnahmen sowie Verhaltensänderungen der Marktteilnehmer ließen fundamentale Strukturveränderungen auf internationalen Finanzmärkten entstehen. An die Stelle längerfristig angelegter Beziehungen zwischen Gläubigern und Schuldnern treten im internationalen Finanzgeschäft zunehmend anonyme, teils spekulative und kurzfristige Varianten des Kapitalaustausches in Form spezifischer Produkte, die weltweit und ganztägig handelbar sind. Mit den Handelsaktivitäten werden auch Finanzrisiken unterschiedlicher Marktsegmente und Zeitzonen miteinander vernetzt.

Der hochgradig mobile und eigendynamische Charakter von Finanztransaktionen ist es, der in aller Regel für die Kapitulation der Politik gegenüber der Ökonomie verantwortlich gemacht wird (vgl. etwa S. Strange 1996; 1998). Immer wiederkehrende Krisen internationalen Ausmaßes wie die lateinamerikanische Verschuldungskrise der frühen Achtzigerjahre oder der Zusammenbruch einiger asiatischer Volkswirtschaften im Jahr 1997/1998 scheinen diese Einschätzung zu bestätigen. Ihre gewachsene Mobilität erlaubt es den regulierten Marktakteuren im Prinzip, sich hohen und für sie damit teuren Sicherheitsauflagen durch Abwanderung aus dem heimischen Territorium („exit“) zu entziehen oder auf deren Absenkung zu drängen („voice“) (vgl. hierzu A. Hirschman 1970). Nationalstaaten und deren Zentralbanken agieren im Krisenfall allenfalls als letzte Refinanzierungsinstanz („lender-oflast-resort“), die die offenen Rechnungen des „globalen Casinos“ mit Hilfe von Steuergeldern zu begleichen haben.

Dieser sowohl von Marxisten als auch von Realisten im Lager der internationalen politischen Ökonomie vertretenen These einer „Ökonomisierung der Politik“ im Bereich des Finanzsektors soll in diesem Beitrag nicht völlig widersprochen werden. Gleichwohl wird argumentiert, dass sich die globale Ausdehnung von Finanzmärkten nicht losgelöst vom nationalstaatlichen Zugriff vollzieht. Am Beispiel der Finanzmarktregulierung, einem in dieser Diskussion bislang unterbelichteten Politikfeld zeigt sich, dass Staaten ihr Instrumentarium an Sicherheitsstandards dem jeweiligen Stand der Marktexpansion und den damit anfallenden Regulierungsproblemen flexibel angepasst und dabei schrittweise erweitert haben. War der Nationalstaat in den Achtzigerjahren in erster Linie Implementeur international vereinbarter Sicherheitsstandards, welche nationale Regulierungskulturen weitgehend unangetastet ließen, so ist er in den Neunzigerjahren Teil von „public-private partnerships“ 
der Regulierung von Bankengeschäften, welche wiederum in pluralisierte Abstimmungsnetzwerke auf internationaler Ebene eingebunden sind. Nicht der Rückzug des Nationalstaates ist die Folge, sondern eine Redefinition regulativer Staatlichkeit, welche sich in den Dienst der Stabilisierung des internationalen Finanzsystems stellt.

\section{Bankenregulierung - Norm und Realität auf nationaler Ebene}

Im Finanzsektor ist der Umgang mit ökonomischen Risiken eine Frage, die auch die Politik interessieren muss. Weil Kreditinstitute eine Multiplikatorfunktion auf dem Finanzmarkt einnehmen und Kreditbeziehungen mit in- und ausländischen Kunden unterhalten, kann die Zahlungsunfähigkeit einer Bank auch Unternehmen anderer Branchen und Nationalitäten in deren Möglichkeiten zu Investitionen und zur Schaffung neuer Arbeitsplätze behindern. Die aus solch weit reichenden Kettenreaktionen resultierenden negativen externen Effekte und letztlich Systemrisiken werden im Allgemeinen als normative Begründung für eine staatliche Regulierung des Finanzgeschehens angeführt. Rechtliche Auflagen zur Bildung finanzieller Sicherheitspolster, zur Offenlegung von Daten über die Geschäftspolitik eines Unternehmens sowie Sanktionsmaßnahmen für den Fall der Missachtung solcher Pflichten sind Instrumente, mit denen der Staat ein vorbeugendes Sicherheitsnetz aufspannen könnte. Im Kern geht es dabei um die Vorgabe von Sicherheitsstandards, die dem eigentlichen Krisenfall vorbeugen sollen. Schließlich soll verhindert werden, dass in dieser Situation die nationale Zentralbank und der Staat als Refinanzier (=,lender-of-last-resort“) einspringen müssen und die Lasten privat erzeugter Risiken letztlich dem Steuerzahler aufgebürdet werden (vgl. zu Problemen der Finanzmarktregulierung, J. Kay/J. Vickers 1988 sowie C. Goodhart et al.1999).

Entgegen solch normativer Vorstellungen zeigt ein historischer Überblick, dass der Staat oftmals erst im Zuge einer größeren Finanzkrise Regulierungsaufgaben übernahm. Für die Vereinigten Staaten waren der Börsenkrach im Jahre 1929 und die darauf folgende Weltwirtschaftskrise der Ausgangspunkt einer Neuordnung des amerikanischen Bankwesens. In Deutschland wurden zentralstaatliche Aufsichtskompetenzen über das private Bankwesen nach der Bankenkrise 1932 aufgebaut. In Großbritannien sollte es noch bis 1979 dauern, bevor die Beaufsichtigung des Bankensektors gesetzlich festgeschrieben wurde. Trotz des Vorhandenseins formaler Regulierungskapazitäten war in vielen Ländern das Instrumentarium zur Eindämmung von Finanzrisiken zunächst unterentwickelt - Bankenaufsicht beschränkte sich entweder auf die restriktive Vergabe von Banklizenzen (USA), auf informelle Konsultationen über Geschäftspraktiken zwischen Zentralbank und großen Banken (Großbritannien) oder auf die Durchsicht von Quartalsberichten der privaten Kreditinstitute (Deutschland). Hierfür gab es im Wesentlichen zwei Gründe:

Zum einen spiegelten Regulierungsstruktur und Regulierungsniveau vielfach Elemente der Staatsstruktur, allgemeine Konfliktmuster zwischen Staat und Kapitalseite und auch nationale Kulturen der Regulierung oder Nicht-Regulierung bestimmter 
Sachverhalte wider. In den USA sind die zwischen Bund und Einzelstaaten teils überlappenden, teils getrennten Regulierungskompetenzen Spiegelbild eines föderalistischen Staates. Eine derartig fragmentierte Zuständigkeitsstruktur in Fragen der Bankenregulierung erfordert Koordinationsleistungen, die Effizienzverluste im Regulierungsalltag mit sich bringen können. Die für Großbritannien charakteristische Kultur der Nicht-Einmischung in private Belange prägte lange Zeit auch die Bankenregulierung. Klientelistische Beziehungen zwischen Zentralbank und der Elite der „City of London“ ersetzten eine an formalen Regeln und Sanktionen orientierte Form der Bankenaufsicht. In Deutschland wiederum beherrschten korporatistische Muster weitgehender Delegation von Regulierungsaufgaben auf die Bankenverbände die nationale Regulierungspraxis. Der Preis eines solchen Modells der „Staatsentlastung“ war eine relative Unkenntnis der staatlichen Aufsichtsbehörden über die tatsächlichen Probleme des Bankengeschäftes (vgl. W. Coleman 1996 zu den unterschiedlichen nationalen Regulierungsstrukturen und -traditionen).

Ein zweiter zentraler Grund für die Unterentwicklung des regulativen Instrumentariums zur Eindämmung von Finanzrisiken liegt darin, dass die im Kreise westlicher Industriestaaten vereinbarte Nachkriegsordnung von Bretton-Woods (1944) den Wettbewerb auf Finanzmärkten und damit auch die Möglichkeiten risikoreicher Engagements beschränkte. Durch die Leitwährungsfunktion des Dollar und die Festschreibung stabiler Wechselkurse unterblieb die Möglichkeit, auf Zins- oder Wechselkursdifferenzen zu spekulieren oder Finanzinnovationen zu entwickeln, um sich gegen diese abzusichern. Kapitalverkehrskontrollen behinderten den Kapitalfluss hin zu möglicherweise attraktiveren Anlagemöglichkeiten im Ausland. Auf heimischen Märkten fungierten funktional segmentierte Bankensysteme (etwa die Trennung zwischen dem Kredit-/Einlagengeschäft und dem Investmentbanking) wie in den USA, Zinsbindungen und Kapitalsteuern (Deutschland) oder hochregulierte Börsenmärkte (Großbritannien) gewissermaßen als Ertrags- und damit auch als „Risikopuffer“ - ein breit gefächertes Instrumentarium der Risikoabsicherung erschien daher zunächst überflüssig. Diese Situation sollte sich mit der Expansion des Marktes über nationale Grenzen hinweg ändern.

\section{Die Befreiung des Marktes - Von nationalen Märkten zur Internationalisierung}

Gewissermaßen „,im Schatten“ des Nachkriegskonsenses wechselseitiger Abschottung nationaler Finanzmärkte kam es in den Sechzigerjahren zu ersten Schritten der Internationalisierung von Wirtschaft und damit auch von Finanzgeschäften. Deutsche Banken folgten ihren multinationalen Firmenkunden ins Ausland, bauten Repräsentanzen an den wichtigsten Finanzzentren auf und gingen Minderheitsbeteiligungen an Geschäftsbanken in Entwicklungsländern ein.

Seit den frühen Sechzigerjahren Jahren stellten die Euromärkte für international operierende Banken eine wichtige „exit“-Option aus dem System national geschlossener Finanzmärkte dar. Bei Euromärkten handelt es sich um internationale Märkte, 
auf denen Fremdwährungsgeschäfte außerhalb des Ursprungslandes der betreffenden Währung getätigt werden konnten. Diese Möglichkeit wurde mit der Einführung der Konvertibilität von Währungen zwischen wichtigen westeuropäischen Ländern im Jahre 1958 geschaffen. Der Begriff „Euro“-Markt charakterisiert einerseits die dort gehandelten Währungen, zu denen neben dem US-Dollar auch die Deutsche Mark, der Schweizer Franken, das Britische Pfund oder der Französische Franc zählten. Zum anderen waren die am Euromarkthandel beteiligten Banken vielfach in europäischen Finanzzentren wie London, Luxemburg oder Zürich ansässig. Gleichwohl unterlag das Euromarktgeschäft weder der Aufsicht einer nationalen Bankenaufsichtsbehörde noch den Eingriffen einer Zentralbank. Dies lag nicht zuletzt daran, dass Zentralbanken und Regierungen die rapide Expansion dieses Marktes jenseits der nationalen Marktbeschränkungen im eigenen Interesse tolerierten. Großbritannien, Luxemburg oder die Schweiz profitierten als Sitzländer vom Eurohandel durch Prestigegewinn ihrer nationalen Finanzplätze und ein hohes Steueraufkommen der dort ansässigen Banken. Die USA und auch westeuropäische Staaten nutzten den Euromarkt zur Finanzierung ihrer im Zuge der Ölkrise 1973 anwachsenden Zahlungsbilanzdefizite. International operierende Großbanken nutzten das Euromarktgeschäft dazu, nationale Zinsbindungen, Kapitalsteuern oder festgelegte Provisionen für Kapitalvermittler zu umgehen. Im Unterschied zu den heimischen Finanzmärkten stellten die Euromärkte eine hochwettbewerbliche und renditeträchtige Umgebung dar, in der Profite durch große Handelsvolumina, mengenabhängige Gebühren und verhandelte Handelsprovisionen entstanden (P. Cerny 1994: 192). Zudem experimentierten die Händler dort mit Finanzinnovationen jenseits herkömmlicher Darlehen oder festverzinslicher Wertpapiere. Es existierte bereits eine Vielzahl neuer Instrumente wie „revolvierende“ Kredite oder „Zinsswaps“. Banken, Wertpapierhändler und Anleger lernten auf diesem Markt insofern frühzeitig den Umgang mit (risikoreichen) Finanzoperationen, welche die spätere Globalisierung des Finanzgeschäftes entscheidend vorantreiben sollten (vgl. zum Euromarkt, G. Junne 1976; E. Storck 1995).

In den Siebzigerjahren bereiteten politische Maßnahmen den Boden für eine weitere Marktexpansion. Der Zusammenbruch des Bretton Woods-Systems und der Übergang zu flexiblen Wechselkursen im Jahre 1973 waren der Auftakt zu einer Welle der Liberalisierung von Finanzmärkten. Die USA trieben den Deregulierungsschub erheblich voran, um ihre durch Vietnam-Krieg und umfangreiche staatliche Förderprogramme massiv anwachsenden Haushaltsdefizite mit ausländischem Kapital zu finanzieren. 1974 wurden Kapitalkontrollen abgebaut und Preiskartelle an der New Yorker Börse aufgehoben. 1979 folgte Großbritannien mit der Aufhebung des vierzig Jahre alten Systems von Kapitalverkehrskontrollen und mit der Liberalisierung der Londoner Börse (Big Bang) 1986 (vgl. zum Liberalisierungsprozess, E. Helleiner 1994).

Die Liberalisierung des Kapitalverkehrs wurde in Europa Teil des Binnenmarktprogramms und im Rahmen der „Einheitlichen Europäische Akte“ (EEA) als bis Ende 1992 zu erreichendes Ziel im EWG-Vertrag fixiert. Die europäischen Staaten verpflichteten sich dem Abbau aller Kapitalverkehrsbarrieren (Richtlinie 88/361/ 
$E W G$ ) und erlaubten europäischen Kreditinstituten, Wertpapierhandelsunternehmen und Börsen, sich europaweit zu betätigen. Im Rahmen der Zweiten Bankrechtskoordinierungsrichtlinie (89/646/EWG) und Wertpapierdienstleistungsrichtlinie (93/ 22 / $E W G$ ) erhielten europäische Finanzinstitute mit der Erteilung ihrer Geschäftslizenz durch die heimatliche Aufsichtsbehörde automatisch den Europa-Pass.

Banken nutzten ihre neugewonnenen Freiheiten dazu, sich neue Geschäftsfelder zu erschließen, die höhere Renditen versprachen, aber auch Geschäftsrisiken neuer Art darstellten. Zunächst lagen diese wesentlich im internationalen Kreditgeschäft mit anderen Staaten, insbesondere mit Schwellenländern. ${ }^{1}$ Die Ausweitung des Bankengeschäftes in neue risikoreiche Geschäftsfelder ging jedoch zunächst nicht mit der Aufstockung von Sicherheitspolstern einher. Vielmehr nutzten Kreditinstitute ihr ausgebautes Netz an Auslandsniederlassungen dazu, sich regulativen Auflagen ihrer heimischen Aufsichtsinstanzen zu entziehen: deutsche Institute (Bankhaus Herstatt) bauten etwa Kreditpyramiden in Luxemburg auf, um gegenüber der deutschen Aufsicht das wahre Ausmaß ihrer Geschäftsrisiken zu verschleiern und auf diese Weise Kapitalrücklagen einzusparen. Amerikanische Banken (wie die Franklin National Bank of New York) nutzten hingegen ihre Filialen in London ${ }^{2}$ zum Aufbau risikoreicher Kreditportfolios und auch dazu, sich am Euromarkt in Devisenspekulationen zu engagieren.

Im Zuge der lateinamerikanischen Verschuldungskrise wurden Regulierungsdefizite offensichtlich. Als sich zu Beginn der Achtzigerjahre Brasilien, Mexiko, Argentinien und Venezuela für zahlungsunfähig erklärten, waren davon vorwiegend amerikanische Banken betroffen. Zusammen mit anderen Regierungen initiierten die USA eine Finanzhilfe des Internationalen Währungsfonds (IWF), welche auf heimischem Boden jedoch heftig umstritten war (W. Reinicke 1995: 143). Die internationale Staatengemeinschaft fungierte somit als „lender-of-last resort“ und übernahm zumindest einen Teil der Kosten privat erzeugter Risiken. Damit war eine Situation entstanden, die gemäß allgemeiner Regulierungsphilosophie immer verhindert werden sollte.

1 Zwischen 1965 und 1980 wuchs das internationale Kreditgeschäft der Banken jährlich durchschnittlich um 25-30 Prozent; lagen die internationalen Verpflichtungen der großen Banken 1965 lediglich 55 Mrd. US-Dollar, so stiegen sie 1981 bis auf 2200 Mrd. US-Dollar an (OECD 1983: 16).

2 Zwischen 1963 und 1972 stieg die Zahl der Auslandsniederlassungen amerikanischer Banken von 150 auf über 650 (OECD 1983: 55) 


\section{$4 \quad$ Internationalisierung und Regimebildung auf zwischenstaatlicher Ebene}

Mit zunehmender Internationalisierung des Finanzgeschäftes bildete sich seit Mitte der Siebzigerjahre ein internationales Kooperationsnetzwerk zwischen Notenbankgouverneuren und nationalen Organen der Bankenregulierung heraus. Einigkeit bestand darüber, dass es nicht darum gehen könne, eine internationale Refinanzierungsinstanz aufzubauen, welche im Krisenfall die Verpflichtungen der Institute übernehmen und auf diese Weise deren Geschäftsrisiken absorbieren solle; zu groß erschien die Gefahr, hierdurch die Risikobereitschaft der Banken unnötig zu steigern (=,moral hazard“; siehe OECD 1983: 109). Multilaterale Kooperation zielte vielmehr darauf, die Reichweite von Regeln der Risikobegrenzung über das jeweilige nationale Territorium hinaus auszudehnen und Banken auf diese Weise die Möglichkeit zu nehmen, sich regulativen Auflagen zu entziehen. Ein im Kreis westlicher Industrieländer vereinbartes Sicherheitsnetz sollte den Konkurs- und damit auch Ernstfall für die jeweiligen nationalen Aufsichtsbehörden verhindern.

Diese Überlegungen führten 1974 zur Einrichtung des Basler Ausschusses für Bankenaufsicht (nach seinem damaligen Vorsitzenden Peter Cooke, auch CookeKomitee genannt). Hierbei handelt es sich um ein Expertengremium von Notenbankgouverneuren und Aufsichtsorganen der G10-Länder sowie der Schweiz, welches der Bank für Internationalen Zahlungsausgleich in Basel angelagert ist. Der Ausschuss lässt sich als internationales „Regime“ (i.S. von S. Krasner 1983) oder auch als Kern einer sich herausbildenden „epistemic community“ (E. Haas 1992) in der internationalen Bankenregulierung charakterisieren, denn die im Kreise von Fachleuten erarbeiteten Empfehlungen ohne Rechtscharakter tragen zur Schaffung eines internationalen Ordnungsrahmens insofern bei als die Regimebeschlüsse von den beteiligten Staaten umgesetzt werden und zudem als Vorgaben für die Richtlinien der Europäischen Union in diesem Feld dienen - als solche erfordern sie dann zwingend eine Umsetzung in nationales Recht.

Bis in die Achtzigerjahre hinein folgte jeder Finanzkrise internationalen Ausmaßes eine Verfestigung zwischenstaatlicher Kooperation, die mit immer weiter gehenden Standards der Risikobegrenzung einherging: im Basler Konkordat von 1975 legte man die Zuständigkeit der Heimatbehörde als Aufsichtsmaxime fest, ergänzte diese aber durch die Verpflichtung zum regelmäßigen Informationsaustausch mit der Aufsicht des Gastlandes. 1979 wurde verfügt, dass multinational tätige Banken in ihrer Bilanz auch die im Ausland getätigten Geschäfte zu berücksichtigen und deren Risiken abzusichern hatten (konsolidierte Überwachung) (E. Kapstein 1994).

Europäische Richtlinien vollzogen Basler Prinzipien der Heimatlandkontrolle und der Kooperation von Aufsichtsorganen 1977 nach. In der Ersten Bankenkoordinierungsdirektive aus dem Jahre 1977 (77/780/EWG) wurde zudem die Gründung eines Ausschusses gefordert, der die weitere Harmonisierung von Regeln der Risikoabsicherung vorbereiten sollte. Nationale Zentralbanken, Aufsichtsbehörden und Finanzministerien legten - im Gegensatz zur Europäischen Kommission - 
Wert darauf, ein Mitspracherecht bei der Vorbereitung weiterer Harmonisierungsschritte zu haben. Aus diesem Konflikt ging die Gründung des Beratenden Bankenausschusses als intergouvernementalem Gremium hervor (siehe auch K.-F. Cordewener 1990). Nach und nach entwickelte sich dieser Ausschuss zum zentralen Beratungs- und Entscheidungsgremium in Fragen der europäischen Bankrechtsharmonisierung, von dem Gesetzesinitiativen ausgingen oder Kommissionsvorschläge in einem sehr frühen Stadium diskutiert werden. 1983 folgte die EU mit der Ersten Konsolidierungsrichtlinie (83/350/EWG) dem Basler Vorstoß zur konsolidierten Überwachung und legte damit ausländischen Tochterbanken die „Zügel“ der heimatlichen Aufsicht an.

Die lateinamerikanische Verschuldungskrise rückte zu Beginn der Achtzigerjahre das Problem der Absicherung internationaler Banken gegen den Ausfall großer Schuldner in den Mittelpunkt der Regulierungsaktivitäten. Nicht nur amerikanische Banken waren unterkapitalisiert und von der Krise betroffen; auch im Ausland sanken die Kapitalrücklagen international tätiger Banken stetig ab. Die heterogenen nationalen Regulierungskulturen erschwerten zunächst internationale Standardisierungsmaßnahmen. Nach mehrjährigen, konfliktreichen Beratungen auf Basler Ebene und einem drohenden Alleingang der USA und Großbritannien wurde im Jahre 1988 die Basler Eigenkapitalempfehlung beschlossen. Darin legten die Länder der G10-Gruppe erstmals einen quantitativen Mindeststandard für die Eigenkapitalausstattung international tätiger Banken fest. Mit dieser Eigenkapitalquote bestand erstmals eine internationale Vereinbarung zur Risikobegrenzung im Finanzsektor, die das bisherige Regulierungsniveau zumindest einiger Mitgliedsstaaten (USA, Japan) überstieg und deshalb als der bislang größte Erfolg der Komiteegeschichte gilt (vgl. zur Geschichte dieser Eigenkapitalempfehlung, E. Kapstein 1992; S. Lütz 1999b). Wie auch in den Siebzigerjahren folgte die Europäische Union den Basler Vorgaben und unternahm mit der Eigenmittel-Richtlinie (89/299/EWG) und der Solvabilitätskoeffizienten-Richtlinie (89/647/EWG) vom Dezember 1989 eigene Schritte in Richtung risikobasierter Kapitalunterlegung.

Ein internationaler Standard der Risikobegrenzung war aus zwei Gründen vorteilhaft: eine zumindest im Kreis westlicher Industrieländer vereinbarte Kapitalquote entzog den Banken „Exit-Optionen“ und verhinderte auf zwischenstaatlicher Ebene einen Unterbietungswettlauf. Zudem erwies sich ein internationaler Standard als „autonomieschonend“ (vgl. zu diesem Punkt auch F. Scharpf 1995), weil nationale Regulierungspraktiken weitgehend unangetastet blieben - so wurde die Kapitalquote in Großbritannien nach wie vor zwischen der Bank of England und einzelnen Banken informell festgelegt, während in Deutschland Aufsichtsamt und Bankenverbände in korporatistischer Manier eine branchenweite Norm bestimmten. Voraussetzung einer standardisierten Form des Risikomanagements war jedoch eines Risiken mussten berechen- und quantifizierbar und ihre kapitalmäßige Absicherung daher verhandelbar sein. In den Neunzigerjahren erodierten diese Bedingungen zusehends. 


\section{$5 \quad$ Die Expansion des Marktes - Von der Internationalisierung zur Globalisierung}

Seit Mitte der Achtzigerjahre zeichnet sich eine schrittweise Globalisierung des Finanzgeschäftes ab. Hierbei handelt es sich um mehr als um eine bloße territoriale Ausweitung finanzwirtschaftlicher Aktivitäten. Die Besonderheit dieser neuen Entwicklungsphase liegt in Strukturveränderungen im internationalen Finanzgeschäft, die neue Herausforderungen für Regulierungsaktivitäten darstellen.

Zu den wichtigsten Strukturveränderungen zählt die Aufwertung des Geschäftes mit dem Handel von Wertpapieren gegenüber dem klassischen Kreditgeschäft (= Verbriefung oder „securitization“ von Finanzbeziehungen, siehe hierzu OECD 1995). Großunternehmen, die als kreditwürdig gelten, ziehen es vor, sich durch die Ausgabe von Wertpapieren (Aktien, Anleihen) selbst an internationalen Kapitalmärkten zu finanzieren, statt sich auf die für sie eher teure Kreditbeziehung zu ihrer Hausbank zu verlassen. International tätige Banken agieren eher als Wertpapierhändler denn als Kreditgeber und erzielen einen immer größeren Teil ihrer Erträge mit Provisionseinnahmen statt mit Zinsgewinnen. Finanzbeziehungen auf internationalen Kapitalmärkten sind immer weniger standardisiert, eher kurzfristig angelegt und anonymisiert.

Institutionelle Anleger wie Pensionsfonds, Investmentfonds und Versicherungen avancierten Ende der Achtzigerjahre zu den treibenden Kräften dieses Strukturwandels (vgl. J. Huffschmid 1999: 82-88). Ihre Anlagetätigkeit besteht darin, eine Mischung (Portfolio) aus Vermögenswerten zusammenzustellen, deren Rendite (etwa Dividenden) möglichst hoch ist. Daraus ergibt sich eine prinzipiell eher kurzfristige Anlagestrategie als die der Banken. Institutionelle Anleger sind bestrebt, ihre Wertpapierbestände nach Ländern, Regionen und Branchen zu diversifizieren. Auf ihrer weltweiten Suche nach den renditeträchtigsten Anlagemöglichkeiten sind sie zudem an immer neuen Produktinnovationen interessiert, die es erlauben, auf Zinsoder Wechselkursdifferenzen zu spekulieren oder sich gegen diese abzusichern.

Das Interesse von Anlegern, Unternehmen und Banken, sich gegen Wechselkursrisiken zu wappnen, ließ den Devisenhandel im letzten Jahrzehnt rapide anwachsen (vgl. hierzu J. Huffschmid 1999: 43-48). Immer neue Finanzinnovationen, die allein auf Erwartungen von Preisänderungen einzelner Devisen basieren, also wie im Fall von Derivaten im Kern „Wetten auf die Zukunft“ sind, erhöhen den ausschließlich spekulativen Anteil der weltweiten Kapitalflüsse und entkoppeln die internationalen Finanzströme zunehmend von der Realwirtschaft. Weil man bei geringem Kapitaleinsatz große Geschäfte abschließen kann, steigen die im Krisenfall betroffenen Kapitalvolumina erheblich.

Fortschritte in der Informations- und Kommunikationstechnik erlauben einen ganztägigen, weltweiten Handel von Finanzprodukten. Dieser findet statt an regulierten Börsenmärkten, in privaten, elektronischen Netzwerken von Banken und Wertpapierhändlern und mittelfristig zunehmend im Internet. Elektronische Vernetzung steigert die Interdependenzen zwischen unterschiedlichen Marktsegmenten 
und Regionen über den Kreis der westlichen Industrieländer hinaus und lässt die Ansteckungsgefahr, die von lokalen Krisenherden ausgeht, erheblich anwachsen.

Unter Regulierungsgesichtspunkten sind zwei Veränderungen von besonderer Bedeutung: erstens, der Kreis möglicher Risikoträger hat sich über den Bankensektor hinaus erweitert. Mit Wertpapierfirmen, Versicherungen, aber auch Unternehmen und institutionellen Anlegern sind es nicht nur Akteure anderer Marktsegmente, sondern auch zunehmend Private, denen sich ebenfalls die Frage angemessenen Risikomanagements stellt. Zudem hat sich der Bereich betroffener Regionen über das Spektrum entwickelter Industrieländer hinaus ausgedehnt.

Zweitens, Finanzrisiken ergeben sich immer weniger aus Formen der klassischen, eher längerfristig angelegten Kreditbeziehung. Risiken neuer Art sind unmittelbar an die Volatilität von Marktzuständen gebunden, schwanken damit je nach Zins- oder Wechselkursentwicklung und sind insofern nicht mehr einfach berechenbar. In der zurückliegenden Asienkrise schätzten alle Beteiligten die Risiken ihrer dortigen Engagements falsch ein. Banken vergaben, kurz vor Ausbruch der Krise, noch umfangreiche Kredite zur Finanzierung von Immobilienprojekten und dies in einer Situation spekulativ angeheizten Baubooms. Rating-Agenturen wiederum stuften die Bonität von Schuldnerländern wie Korea bis kurz vor dessen Zahlungsunfähigkeit als gut ein (vgl. W. Artopoeus 1998).

Für die internationale Staatengemeinschaft stellt sich der Umgang mit Systemrisiken damit anders dar als noch in den Achtzigerjahren: Ging es damals vornehmlich darum, Marktakteuren durch Vereinbarung kollektiver Regeln Arbitragemöglichkeiten zu entziehen, so ist es nun außerdem die Komplexität und Volatilität des Marktgeschehens, auf die eine neue regulative Antwort gefunden werden muss. Im Unterschied zu den Achtzigerjahren lassen sich Finanzrisiken heute nicht mehr so einfach durch standardisierte Eigenkapitalquoten absichern. Risikostrukturen unterscheiden sich je nach dem jeweiligen Geschäftsportfolio einer Bank erheblich; zudem führt die stärkere Marktgebundenheit der Geschäfte dazu, dass standardisierte Absicherungsmechanismen schnellen Marktveränderungen unterworfen und daher wenig treffsicher sind. Welchen Nutzen besitzt schließlich ein Eigenkapitalstandard, wenn sich dieser stündlich verändern kann?

\section{6 \\ Globalisierung und Ausdifferenzierung der internationalen Regulierungsarchitektur}

Seit Beginn der Neunzigerjahre differenziert sich die internationale Regulierungsarchitektur zweifach aus: das Spektrum der an der Finanzmarktregulierung Beteiligten erweitert sich über den Kreis von Notenbanken und Regulatoren der G7Staaten hinaus (horizontale Dimension). Zum anderen zeichnet sich ein intensiveres Zusammenwirken von Akteuren unterschiedlicher Handlungsebenen (national, international, global) ab (vertikale Dimension). 
Für die Weiterentwicklung von Regulierungsstandards im Kreise westlicher Industrieländer ist in Zukunft die verstärkte Kooperation staatlicher und privater Akteure von zentraler Bedeutung. Diese ist Reaktion auf eine Situation allgemeiner Unsicherheit bei der Risikobegrenzung, in der insbesondere der Staat auf die Regulierungspraxis der Marktteilnehmer angewiesen ist, um möglichst „treffsichere“ Regulierungswege zu finden (vgl. S. Lütz 1999b). Außerdem spricht die Tatsache, dass ein wachsender Anteil des weltweit kursierenden Kapitals privater und zudem spekulativer Natur ist, dafür, Marktteilnehmer auch bei der Weiterentwicklung eines vorbeugenden Sicherheitsnetzes stärker in die Verantwortung zu nehmen als bisher. Zudem versucht eine international immer schlagkräftiger organisierte Bankenlobby, regulative Belange selbst in die Hand zu nehmen. Gruppen wie die Group of Thirty (G30) oder das Institute of International Finance (IIF) schlugen vor, doch einen Ständigen Ausschuss auf globaler Ebene einzurichten, in dem „global players“ zusammen mit Aufsichtsvertretern Leitlinien für die weit gehende Selbstregulierung des internationalen Finanzgeschäftes entwickeln - bislang blieben diese Vorschläge ohne Resonanz auf der staatlichen Seite.

In der Bankenregulierung zeichnet sich jedoch der Übergang zu einem eher qualitativen, auf die Vorgabe von Verfahren und Kontrolle von deren Ergebnissen ausgerichteten Regulierungsansatz ab, welcher im Jahre 2002 den „Basler Akkord“ von 1988 formal ablösen soll (Basler Ausschuss für Bankenaufsicht 1999a). International tätigen Banken wird erlaubt, ihre Geschäftsrisiken unter Rückgriff auf eigene, mathematische Modelle bzw. interne oder externe Ratings zu spezifizieren und die zur Risikoabsicherung erforderlichen Kapitalrücklagen selbst zu berechnen. Weil diese Kapitalquoten nicht nach groben Kategorien standardisiert, sondern auf die besondere Geschäfts- und damit auch Risikosituation einer Bank abgestimmt sind, verspricht man sich von diesem Vorgehen eine größere Genauigkeit der Risikoerfassung und -absicherung. Ob der Rückgriff auf das Urteil privater Ratingfirmen ein realistischeres Bild von Schuldnerrisiken vermitteln kann, erscheint allerdings fraglich - schließlich unterlag die Ratingbranche in den zurückliegenden Krisen denselben Fehleinschätzungen wie alle anderen Akteure auch.

Die Rolle der staatlichen Aufsicht wird zukünftig in der permanenten „Evaluation der Selbstevaluation“ global tätiger Banken bestehen. Prinzipiell kann dies im Einzelfall auch höhere Anforderungen an die Bildung von Kapitalpuffern bedeuten. Der Nationalstaat in Gestalt der nationalen Bankenaufsicht wandelt sich insofern vom bloßen Überwacher der Einhaltung einmal gesetzter Regeln hin zum Bankbetreuer, welcher Prozeduren und Ergebnisse privaten Risikomanagements zu kontrollieren und letztlich „abzunehmen“ hat.

Zur Pluralisierung des Akteurspektrums zählt auch der Versuch der G7-Staaten, bestehende Aufsichtsstandards auf die aufstrebenden asiatischen und lateinamerikanischen Märkte zu übertragen und enger mit den dortigen Aufsichtsbehörden zu kooperieren, denen es oftmals an der politischen Unabhängigkeit zur Ausübung ihrer Aufsichtstätigkeit, an Ressourcen wie auch an der erforderlichen Qualifikation fehlt. Neue Kooperationsbeziehungen wurden auch mit Aufsichtsbehörden des 
Wertpapier- und Versicherungssektors aufgebaut, deren Märkte und Risiken zunehmend zusammenwachsen.

In vertikaler Richtung ist ein intensiveres Zusammenwirken unterschiedlicher politischer Handlungsebenen in Regulierungsfragen zu beobachten. Fragen finanzwirtschaftlicher Systemstabilität wurden erheblich politisiert, was sich in wachsenden Aktivitäten der G7-Gruppe äußert. Auf den regelmäßigen Gipfeltreffen der Finanzminister und ihrer Referenten werden von den Aufsichtsinstanzen Berichte über Fortschritte ihrer Aufsichtstätigkeit angefordert und neue Aufgabenstellungen formuliert. Seit 1998 rückt die Suche nach institutionellen Pfeilern einer stabilen internationalen Finanzarchitektur in den Mittelpunkt des Interesses der G7-Gruppe. Auf dem Gipfel in Washington im Oktober 1998 beauftragte man den ehemaligen Bundesbankchef Tietmeyer, institutionelle Antworten auf die jüngsten Finanzkrisen zu erarbeiten. Dies war der Hintergrund für die Gründung des Forums für Finanzstabilität im Februar 1999 (vgl. hierzu H. Tietmeyer 1999 und Financial Stability Forum 1999).

Hierbei handelt es sich um einen dauerhaften Roundtable von Vertretern der an der Stabilität von Finanzmärkten mitwirkenden Instanzen. Das Gremium unterscheidet sich von bestehenden Foren insofern, als es nationale Aufsichtsinstanzen und Zentralbanken mit Repräsentanten internationaler Organisationen (IWF, Weltbank, BIZ und OECD) und Regulierungsgremien (Basler Komitee, Wertpapier-und Versicherungsaufsicht) zusammenführt und den Kreis beteiligter Staaten über den G7-Klub hinaus erweitert (so sind auch Hong Kong, Singapur und Australien dort repräsentiert). Private Akteure sind nicht vertreten, sollen jedoch zu Regulierungsfragen konsultiert werden. Ziel der Aktivitäten ist letztlich die Formulierung eines flexiblen Ordnungsrahmens für den globalen Finanzmarkt, die Schaffung einer internationalen Finanzmarktverfassung „,von unten“ (Handelsblatt vom 22.2.1999: 2).

\section{Schlussfolgerungen}

Seit der Entstehung erster Finanzkrisen hat der Staat regulativ-korrigierend in das Geschehen auf nationalen Finanzmärkten eingegriffen, um zukünftigen Krisenerscheinungen vorzubeugen. Daran hat sich auch unter Globalisierungsbedingungen nichts geändert. Ebenso wenig wurden Standards der Risikobegrenzung in den letzten zwei Jahrzehnten abgesenkt - zumindest nicht im Kreise der westlichen Industriestaaten. Vielmehr haben diese ihr Regulierungsinstrumentarium dem jeweiligen Stand der Marktexpansion angepasst und auf diese Weise weiterentwickelt. Nicht der Rückzug des Nationalstaates, sondern die Redefinition seiner Interventionsformen ist die Folge (vgl. auch S. Lütz 1997 zur Situation in der Kapitalmarktregulierung).

Mit der Internationalisierung von Finanzgeschäften veränderte sich jedoch die Regulierungssituation insofern, als Krisenvorbeugung jetzt ein transnationales Gemeinschaftsgut darstellt, welches nur durch kollektives Handeln der internationalen 
Staatengemeinschaft bereitgestellt werden kann. In den Achtzigerjahren zielte diese kollektive Strategie auf Verhandlung eines Standards, welcher international tätigen Banken Arbitragemöglichkeiten entzog und sich auf nationaler Ebene als „implementationsfreundlich“ erwies. Zumindest im Kreis der westlichen Industrieländer kam es seitdem zu einer Anhebung der Eigenkapitalquoten internationaler Banken.

In den Neunzigerjahren wurde die Komplexität und Volatilität der jetzt stärker vermarktlichten und anonymisierten Formen von Finanzbeziehungen zu einem zentralen Problem der Finanzmarktregulierung. Nationalstaaten reagierten darauf mit einem flexiblen Regulierungsansatz, der private Selbstregulierung mit stetiger Überwachung und Evaluation durch den Nationalstaat koppelt und diese interaktive Beziehung in internationale, jetzt pluralisierte Netzwerke der Abstimmung einbettet. Institutionen an der Schnittstelle von politischen Ebenen und unterschiedlichen Akteurgruppen wie das Forum für Finanzstabilität übernehmen neue Koordinationsaufgaben.

Prinzipiell erscheinen solch offenen und auf Diskurs mit allen betroffenen Akteurgruppen setzenden Institutionen dazu geeignet, sich in schnell wandelnden Umwelten zu behaupten und kollektive Unsicherheit zu reduzieren. So haben Studien sozialer Netzwerke aufgezeigt, dass längerfristige Kooperationsbeziehungen zu Präferenzänderungen der Beteiligten, Lerneffekten und neuen Problemlösungen führen können (vgl. etwa W. Powell 1996). Umso aufwendiger erscheint jedoch ihr Management: in der Finanzmarktregulierung geht es zum einen darum, heterogene Kulturen der Regulierung unterschiedlicher Regionen und Marktsegmente in Prozesse der Standardisierung einzubeziehen. Im Umgang mit Schwellenländern kann dies den Ausgleich eines Entwicklungsgefälles bei der Entwicklung von Markt und Aufsichtsinfrastruktur erfordern. Die Kooperation mit privaten Akteuren birgt für die Staatenseite zudem die Gefahr, sich in Ermangelung besseren Wissens allzu leicht auf ein niedrigeres Regulierungsniveau zu begeben und auf diese Weise für private Zwecke instrumentalisiert zu werden („capture“).

Ein „Bottom-up-Ansatz“, welcher die Spielregeln einer neuen globalen Finanzmarktverfassung von unten her aufbauen will, stellt neue Anforderungen an die heimische Aufsichtsverwaltung. Eine in den westlichen Industriestaaten mehr und mehr interaktive und know-how-intensive Form der Bankenaufsicht fordert vom Aufsichtspersonal Qualifikationen, die es erlauben, das Bankengeschäft und dessen Risiken zu verstehen - dies ist für die durch das Juristenmonopol und eine formale Regulierungskultur gekennzeichnete deutsche Verwaltung eine besondere Herausforderung (vgl. zum Umbau auf nationaler Ebene, S. Lütz 1999a). Dass aus enger Zusammenarbeit zwischen Aufsicht und „global players“ auf nationaler Ebene Impulse hervorgehen, die auf internationaler Ebene wiederum zu neuen Standards oder funktionalen Äquivalenten im Sinne vereinbarter Mindestanforderungen an die nationale Aufsichtstätigkeit führen, ist zukünftig von entscheidender Bedeutung. Ansonsten bestünde die Gefahr, dass sich etwa in der Bankenregulierung, nationale Lerngemeinschaften aus „global players“ und Aufsichtsinstanzen in neue Unterbietungswettläufe hineinbegeben und wiederum das kollektive Dilemma erreicht wäre, 
das man in den Siebzigerjahren durch zwischenstaatliche Kooperation zu überwinden suchte.

Abschließend sei einem übertriebenen „Steuerungsoptimismus“ vorgebeugt - die Gefahr von Finanzkrisen kann auch durch ein regulatives Sicherheitsnetz nicht völlig unterbunden werden. Der Markt lässt sich nicht berechnen, technologischer Fortschritt und dessen Risiken lassen sich nicht vollständig kalkulieren. Betont sei außerdem, dass Regulierung nur ein Teilelement einer zukünftigen internationalen Finanzarchitektur sein kann. Im Fall der Asienkrise waren es falsche Wechselkurspolitik (etwa die Bindung des heimischen Wechselkurses an den der Hauptinvestoren) und der schnelle Rückzug großer Volumina kurzfristigen und teils spekulativen Kapitals durch westliche Anleger, welche die Kette von Währungsabwertung, Verfall der Aktienkurse und Bankenzusammenbrüchen in Gang setzten. Eine adäquate Währungspolitik und ggf. auch Maßnahmen zur Beeinflussung spekulativer Devisenströme sowie Prozeduren der Krisenbewältigung müssen weitere Pfeiler dieser internationalen Architektur sein. Hierbei gilt es, eine Vielzahl unterschiedlicher Interessen - die der westlichen Industriestaaten, der Schwellenländer und die privater Marktteilnehmer politisch auszubalancieren.

\section{Literatur}

Artopoeus, Wolfgang (1998): Kreditrisiko - Erfahrungen und Ansichten eines Aufsehers. Vortrag beim Symposium „Kreditrisiko“ der Deutschen Bundesbank am 24. November 1998 [http: / / www.bakred.de/texte/praes/r_241198.htm]

Basler Ausschuss für Bankenaufsicht (1999a): Konsultationspapier zur Neuregelung der angemessenen Eigenkapitalausstattung, Nr. 23. Basel: Bank für internationalen Zahlungsausgleich

Basler Ausschuss für Bankenaufsicht (1999b): Capital Requirements and Bank Behaviour: The Impact of the Basle Accord. Working Paper No.1. Basel: Bank für internationalen Zahlungsausgleich

Cerny Philip G. (1994): Money and Power: The American Financial System From Free Banking to Global Competition. In: Thompson, Grahame (Hrsg.) (1994): Markets. Walton Hall / Milton Keynes: The Open University: 175-213

Coleman, William D. (1996): Financial Services, Globalization and Domestic Policy Change: A Comparison of North America and the European Union. London: Macmillan

Cordewener, Karl-Friedrich (1990): Internationale Gremien für Bankaufsichtsfragen. In: Die Sparkasse 11. 505-511

Financial Stability Forum (1999): Background Brief Made to the Press at the 2nd Meeting of the Financial Stability Forum on 15 September 1999 [http://www.bis.org/press/p990916.htm]

Goodhart, Charles et al. (1998): Financial Regulation. Why, How and Where Now? London: Routledge

Haas, Peter M. (1992): Introduction: Epistemic Communities and International Policy Coordination. In: International Organization (1): 1-36

Handelsblatt vom 22.2.1999: Sünder werden vom Markt bestraft. 2

Helleiner, Eric (1994): States and the Reemergence of Global Finance. From Bretton Woods to the 1990s. Ithaca, N.Y.: Cornell University Press

Hirschman, Albert O. (1970): Exit, Voice and Loyalty. Cambridge, MA.: Cambridge University Press

Huffschmid, Jörg (1999): Politische Ökonomie der Finanzmärkte. Hamburg: VSA-Verlag

Junne, Gerd (1976): Der Eurogeldmarkt. Frankfurt a.M. / New York: Campus

Kapstein, Ethan B. (1992): Between Power and Purpose: Central Bankers and the Politics of Regulatory Convergence. In: International Organization 43. 323-347

Kapstein, Ethan B. (1994): Governing the Global Economy: International Finance and the State. Cambridge, MA.: Harvard University Press

Kay, John/Vickers, John (1988): Regulatory Reform in Britain. In: Economic Policy 8. 285-351 
Krasner, Stephen D. (Hrsg.) (1983): International Regimes. Ithaca, NY.: Cornell University Press Lütz, Susanne (1997): Die Rückkehr des Nationalstaates? Kapitalmarktregulierung im Zeichen der Internationalisierung von Finanzmärkten. In: Politische Vierteljahresschrift 38. 475-498

Lütz, Susanne (1999a): Globalisierung und der regulative Umbau des Modells Deutschland - Das Beispiel der Bankenregulierung. In: Brose, Hanns-Georg/Voelzkow, Helmut (Hrsg.) (1999a), Institutioneller Kontext wirtschaftlichen Handelns und Globalisierung. Marburg: Metropolis: 205-233

Lütz, Susanne (1999b): Zwischen „Regime“ und „kooperativem Staat“ - Bankenregulierung im internationalen Mehr-Ebenen-System. In: Zeitschrift für internationale Beziehungen 6. 9-40

Organisation for Economic Cooperation and Development (OECD) (1983): The Internationalisation of Banking. Paris: OECD

Organisation for Economic Cooperation and Development (OECD) (1995): Securitization. An International Perspective. Paris: OECD

Powell, Walter W. (1996): Weder Markt noch Hierarchie: Netzwerkartige Organisationsformen. In: Kenis, Patrick/Schneider, Volker (Hrsg.) (1996), Organisation und Netzwerk. Institutionelle Steuerung in Wirtschaft und Politik. Frankfurt a.M./ New York: Campus: 213-273

Reinicke, Wolfgang H. (1995): Banking, Politics and Global Finance. American Commercial Banks and Regulatory Change 1980-1990. Aldershot/ Brookfield: Edward Elgar

Scharpf, Fritz W. (1995): Autonomieschonend und gemeinschaftsverträglich: Zur Logik europäischer Mehr-Ebenen-Politik. In: The European Yearbook of Comparative Government and Public Administration 1. 89-117

Storck, Ekkehard (1995): Euromarkt: Finanz-Drehscheibe der Welt. Stuttgart: Schäffer-Poeschel

Strange, Susan (1996): The Retreat of the State. Cambridge/New York: Cambridge University Press

Strange, Susan (1998): Mad Money. When Markets Outgrow Governments. Ann Abor: Michigan University Press

Tietmeyer, Hans (1999): Internationale Zusammenarbeit und Koordination auf dem Gebiet der Aufsicht und Überwachung des Finanzmarktes. In: Deutsche Bundesbank, Monatsbericht Mai 1999. 5-14 